\title{
Response to Letter to the Editor: Effects of New Antiplatelet Agents and Aldosterone Receptor Antagonists on Mortality in Patients with Myocardial Infarction
}

\author{
Amjad Abualsuod ${ }^{1}$
}

Published online: 25 August 2015

(C) Springer Science+Business Media New York 2015

\section{Dear Editor,}

Thank you for giving me the chance to respond to Dr. Eyuboglu Comment [1]. He referred to two important points: The use of dual antiplatelet agents and the use of aldosterone receptor antagonists in post myocardial infarction and their effect on mortality. Unfortunately, we did not collect data regarding the distribution of these agents specifically in our study [2]. Thus, we can't say for sure if they were playing a role in the lower mortality observed in the metformin group. This being said, some surrogate markers can be used, with reservation, to address these two points. We had data on Aspirin (the usual second half of the dual antiplatelet regimen) in all the patients in the study. We demonstrated that the associated lower mortality with metformin use persisted after adjustment for aspirin use. In addition, there was no difference in left ventricular ejection fraction on presentation between the three groups and, although the data was not complete, there was no difference at follow up as well. Thus We don't have a reason to believe that there was a difference in the use of diuretics between the groups. We want to stress that this was a retro- spective study with a relatively small number of patients and it should be looked at as a proof of concept study. Thus, additional prospective studies should be performed to clearly identify the effect of metformin in this patient population.

Sincerely,

Amjad Abualsuod, MD

Assistant Professor

Division of General Internal Medicine

UAMS

\section{References}

1. Eyuboglu M. Effects of new antiplatelet agents and aldosterone receptor antagonists on mortality in patients with myocardial infarction. Cardiovasc Drugs Ther. 2015.

2. Abualsuod A, Rutland JJ, Watts TE, Pandat S, Delongchamp R, Mehta JL. The Effect of Metformin Use on Left Ventricular Ejection Fraction and Mortality Post-Myocardial Infarction. Cardiovasc Drugs Ther. 2015;29(3):265-75.
Amjad Abualsuod

amabualsuod@UAMS.edu

Division of General Internal Medicine, UAMS, Litte Rock, AR, USA 